\title{
ON ONE INVERSE PROBLEM OF OPTIMAL PLANNING UNDER UNCERTAINTY IN PREDICTION
}

\author{
Sharif E. Guseynov ${ }^{1,2}$, Jekaterina V. Aleksejeva ${ }^{1}$ \\ ${ }^{1}$ Liepaja University, Latvia; ${ }^{2}$ 'Entelgine" Research \& Advisory Co., Ltd, Latvia \\ sh.e.guseinov@inbox.lv, jekaterina.v.aleksejeva@gmail.com
}

\begin{abstract}
In the present paper we consider an enterprise for production of biogas from animal waste. As it happens with any process, some dangerous waste is generated in the process of biogas production, which usually is stored and neutralized in a special settling tank. The external conditions in which such enterprises operate are such that certain external masses get into the tank, for instance, water entering the reservoir as a result of the floods, precipitation, etc. Consequently, if the reservoir is overfilled, then some of its contents get into the external environment, causing harmful effects both on the human and animal habitat and on the industry itself. In this paper a mathematical model in the form of a single-criterion inverse optimization problem is developed for planning biogas production under conditions of uncertainty, when in addition to the planned production indicators there are also indicators which values are not known a priori and are to be determined as a prediction, taking into account the fact that planning and predictiveindicators are bound by joint constraints in the form of inequalities and equalities.
\end{abstract}

Keywords: optimal planning, prediction uncertainty.

\section{Introduction}

Biowaste arises from a number of human activities, including agriculture, horticulture and industry, broadly falling into one of the following three major categories: faeces/manures, raw plant matter or process waste. Animal origin waste, so-called animal biowaste, such as that contained in sewage and soiled animal bedding contains unabsorbed fats, proteins and carbohydrates, resulting from incomplete digestion of ingested food of animal and plant origin [1]. In addition, abattoir waste would include all of the above and a substantial proportion of fats and protein, derived from the slaughtered animals. Besides, materials excreted by the animal include metabolic breakdown products, such as urea and other small nitrogen-containing materials, for example, partially degraded bile pigments. Live and dead bacteria, normally resident in animal gut, are also present in biowaste and so contribute their own fats, proteins, carbohydrates and nucleic acids. In addition to all the components listed above, biowaste of plant origin will contain cellulose, hemicelluloses and lignin. Biowaste makes up a huge percentage of refuse: between $4080 \div 5100$ million tonnes arise each year in the European Union alone and this is a figure which many authorities suggest increases by between 3-5\% annually [1-4]. Of these astronomic volumes of biowaste, between $1600 \div 2000$ million tonnes arise as agricultural in origin, between $880 \div 1100$ million tonnes consist of garden and forestry waste, between $800 \div 1000$ million tonnes is sewage, $400 \div 500$ million tonnes result from the food-processing industry, $400 \div 500$ million tonnes is municipal solid waste. One of the perspective technologies of producing ecologically pure and persistent energy carrier in the form of biogas is an anaerobic process of fermentation of organic substances derived from animal biowaste. Biogas produced in this way consists of methane $\left(\mathrm{CH}_{4}, 60-65 \%\right)$ and carbon dioxide $\left(\mathrm{CO}_{2}, 35-40 \%\right)$ as well as insignificant amount of impurity of hydrogen $\left(\mathrm{H}_{2}\right)$, hydrogen sulphide $\left(\mathrm{H}_{2} \mathrm{~S}\right)$, ammonia $\left(\mathrm{NH}_{3}\right)$, nitrogen oxides $\left(\mathrm{NO}_{\mathrm{x}}\right)$ and oxygen $\left(\mathrm{O}_{2}\right)$, which total volume is less than $5 \%$. After biogas purification of $\mathrm{CO}_{2}$, biomethane is obtained, which is a complete analogue of natural gas and, therefore, it can be used as an unconventional source of energy. Important advantages of using biogas produced from animal waste are its constant renewability, availability of local sources of raw materials for fuel production, reduction of the greenhouse effect and environmental damage due to the permanently operating system of collecting organic waste, providing environmentally closed (almost) energy system. In addition to it, this technology of biogas production allows to significantly reduce the harmful effects of hazardous components of animal waste (pathogenic microorganisms, helminths, enterococci, staphylococci, pseudomonades, hemophilic bacilli, etc.) on the environment, particularly, on soil and water $[1 ; 5 ; 6]$.

In the present paper we consider an enterprise for production of biogas from animal waste, and we assume that the external conditions, in which the considered enterprise operates, are such that certain external masses get into the tank, for instance, water entering the reservoir as a result of the floods, 
precipitation, etc. Consequently, if the reservoir is overfilled, then some of its contents get into the external environment, causing harmful effects both on the human and animal habitat and on the industry itself [7]. From a mathematical point of view, the considered problem means that it is necessary to construct a mathematical model of optimal planning in uncertainty conditions, when in addition to the planned optimal indicators there are also indicators which values need to be predicted, at that the planning and predictive indicators are related by joint conditions in the form of inequalities and equalities. Here it is necessary to clarify what will be understood by the terms "planning" and "prognostication" in the framework of this work. Speaking of planning, we will imply the process of finding indicators, which vary in a sufficiently wide range, provided that it is possible to put into practice the planned values of the indicators; speaking of prognostication, we will imply the process of finding future values of indicators using their known values in the past, at that we will assume that the pattern of the dynamics of these indicators is either already known or can be determined taking into account some existing factors (for example, developed regulatory requirements, available scientific and technical capabilities, etc.). The following question arises: is it possible to divide the considered into two parts - into the preliminary predictive problem and into the main optimization planning problem? In the general case, such a decomposition of the original complex problem into relatively simple composite tasks will not be correct, because, first, the desired optimal plan depends on the prediction of parameters included in the planning model, and, second, the desired predictions of these parameters depend on the optimal plan that has not been selected yet [8-12]. Since the planning and predictive indicators are connected by joint constraints, the desired values of the predictive indicators must be such that, with the selected prognoses, the optimization model has a solution. Below we propose one of the possible approaches to constructing a mathematical model for the joint finding of a prognosis and a plan in the problem of planning biogas production and prediction of possible ecological and economic consequences of this production.

\section{Formalization and mathematical statement of the general predictive and planning problem}

We introduce the following denotation: $x \in X \subseteq R^{n}$ is the vector of planning indicators; $y \in Y \subseteq R^{m}$ is the vector of predictive indicators; $Y \times X=\{(y, x) \mid y \in Y, x \in X\}$ is Cartesian product of sets $Y$ and $X ; Z=\{z=(y, x) \mid y \in Y, x \in X\} \subseteq Y \times X$ is the set of joint restrictions on the prognosis and plan; $\operatorname{Pl}(x)=\left\{y^{\prime} \in Y \mid\left(y^{\prime}, x\right) \in Z\right\}$ is the set of all possible plans; $\operatorname{Pr}(y)=\left\{x^{\prime} \in X \mid\left(y, x^{\prime}\right) \in Z\right\}$ is the set of all possible prognoses; $\tilde{X}=\left\{x \in X \mid z=(y, x) \in Z, y=y_{f i x} \in Y\right\}$ is the set of plans for a specific prognosis $y=y_{f i x} \in Y ; \quad \tilde{Y}=\left\{y \in Y \mid z=(y, x) \in Z, x=x_{f i x} \in X\right\}$ is the set of predictions for a certain version of plan $x=x_{f i x .} \in X ; F_{p r}(x) \subset \operatorname{Pl}(x), \forall x \in \tilde{X}$ is the set of all feasible for implementation plans when considering version of plan $x ; F_{p l}(y) \subset \operatorname{Pr}(y), \forall y \in \tilde{Y}$ the set of all feasible for implementation plans when considering version of prognosis $y$. Obviously, $F_{p r}(x)$ and $F_{p l}(y)$ are multi-valued mappings with values in $Y$ and $X$, respectively. Following [8], we will call $z=\left.(y, x)\right|_{y \in F_{p r}(x), x \in F_{p l}(y)} \in Z$ a rational choice of predictive-planning indicators both from the point of view of joint restrictions on the prognosis and plan, and from the point of view of the possible implementation in practice of the prognosis and plan. If we are given a point-set mapping

$$
f(y, x)=F_{p r .}(x) \times F_{p l .}(y)=\left\{z^{\prime}=\left(y^{\prime}, x^{\prime}\right) \mid y^{\prime} \in F_{p r .}(x), x^{\prime} \in F_{p l .}(y)\right\},
$$

then the problem of rational choice of the vector $z$ is equivalent to the problem of finding fixed points of the point-set mapping(1) on set $Z$, i.e.

$$
z_{f . p .}=\operatorname{Arg}\left\{Z_{f . p .}\right\}=\operatorname{Arg}\{z \in Z \mid z \in f(z)\} .
$$

where set $Z_{f . p .}$ is called a set of weak solutions of the predictive-planning problem [8].

Let us assume that on $Z$ there is an additional $w(z), z \in Z$. Then the problem 


$$
w(z) \rightarrow \min _{z \in Z}
$$

defines the set of strong solutions of the predictive-planning problem in the sense of the criterion $w(z)$, i.e. $Z_{\text {s.s. }}=\operatorname{Arg} \min \left\{w(z) \mid z \in Z_{\text {f.p. }}\right\}$. Now, having (1)-(3), we can construct (in one of many possible ways) a specific kind of multi-valued mappings $F_{p r}(x)$ and $F_{p l}(y)$. Let us start by constructing a mapping $F_{p l}(y)$ on the set $\tilde{Y}$. Suppose that on the set $Z$ a real function $\varphi(y, x)$ is set, which the decision-maker (deciding on the choice of vector of planning indicators) is trying to minimize for fixed $y=y_{f x x} \in \tilde{Y}$ and with conditions $x \in F_{p l .}(y)$ :

$$
\varphi(y, x) \rightarrow \min _{x \in F_{p l}(y)} .
$$

We write $\left.R(y)\right|_{y=y_{f x t} \in \tilde{Y}}=\operatorname{Arg} \min \left(\varphi(y, x) \mid x \in F_{p l .}(y)\right)$, which can be interpreted as a set of optimal responses for a fixed prognosis $y=y_{f i x} \in \tilde{Y}$ when choosing the planning indicators, and suppose some set of plans $\mathrm{Pl}_{X}$ is given, for whichimplementation of $\left.\mathrm{Pl}_{X} \supset R(y)\right|_{y=y_{f x} \in \tilde{Y}}$ is desirable. Then for $y=y_{f i x} \in \tilde{Y}$ we can define $F_{p l .}(y)=\left.R(y)\right|_{y=y_{f x x} \in \tilde{Y}} \cap \mathrm{Pl}_{X}$. If $\left.R(y)\right|_{y=y_{f x x} \in \tilde{Y}} \cap \mathrm{Pl}_{X} \neq \varnothing$, then vector $y=y_{f i x} \in \tilde{Y}$ is feasible for planning. Problem (4) is an optimal planning problem for a given $y=y_{f x} \in \tilde{Y}$. We should note that in the case when $\mathrm{Pl}_{X} \neq X$, finding feasible vector $y \in \tilde{Y}$ is a complicated problem. Now we will try to construct mapping $F_{p r}(x)$ on set $\tilde{X}$. First of all, we note that, unlike the planning indicator with a known prognosis, the prognosis with the given planning indicators cannot be the result of optimization strong control actions, although formally finding the prognosis may be associated with solving some optimization problem. Now suppose that the external environment is characterized by $N$ states $\left\{S_{j}\right\}_{j=1, N}$, at that $j$-th state is realized with probability $p_{j}$, i.e. $p\left\{S_{j}\right\}=p_{j}, \forall j=\overline{1, N}$. Next, suppose that the predicted vector $y \in Y$ is random when realizing $j$-th state of the external environment, and that for the chosen plan $x \in \tilde{X}$ the value of this predicted vector is $\tilde{y}_{j}(x)$. Let us construct the following set:

$$
\Omega_{\delta}(x)=\left\{y \in \operatorname{Pl}(x)\left|\max _{k=1, N}\right| y_{k}-\sum_{j=1}^{N} p_{j} \cdot \tilde{y}_{j}(x) \mid \leq \delta, \forall \delta>0\right\}, \forall x \in \tilde{X},
$$

which is a product of sets for all kinds of plans $\operatorname{Pl}(x)$ having a $\delta$-neighborhood of mathematical expectation of prognosis for the selected plan $x \in \tilde{X}$. Finally, let us suppose that a certain set of predictions $\operatorname{Pr}_{Y}$ is given, for which meeting of the condition $\Omega_{\delta}(x) \cap \operatorname{Pr}_{Y} \neq \varnothing$ is desirable. Then we can define the mapping $F_{p r}(x)$ as $\Omega_{\delta}(x) \cap \operatorname{Pr}_{Y} \neq \varnothing$. If $\Omega_{\delta}(x) \cap \operatorname{Pr}_{Y} \neq \varnothing$, then vector $x \in \tilde{X}$ is feasible for making a prediction. Based on the procedure for construction of a mathematical model of a general predictive-planning problem outlined in the current section, a mathematical model for determining the optimal plan for the production of biogas from animal husbandry waste with minimizing possible undesirable consequences for the environment will be constructed in the next section.

\section{Modelling of the investigated problem of determining the optimal plan for the production of biogas from animal husbandry waste}

\subsection{Verbal description of the investigated problem and construction of qualitative model}

Since the processes associated with the production of biogas and the disposal of hazardous waste are incurringsome costs, in this paper we assume that, if damage is caused to the external environment (possibly also to the production of biogas itself), then this damage can be measured and estimated in terms of the final output of biogas production, i.e. in profit (damage is deducted from profit). Next, let the external environment conditions $N$ be defined by scenarios $\left\{S_{j}\right\}_{j=1, N}$, which can be carried out 
with some probabilities $\left\{p_{j}\right\}_{j=1, N}: \sum_{j=1}^{N} p_{j}=1$. The studied problem is aimed at finding a production plan and a related prognosis of possible emissions of hazardous industrial waste into the external environment, which results in negative environmental and economic consequences. It is important that the desired plan should take into account the probabilities of implementation of environmental scenarios $\left\{S_{j}\right\}_{j=1, \bar{N}}$ and their predicted environmental and economic consequences: there is no full guarantee that there will be no such emission of hazardous waste from biogas production, since various natural disasters are possible, accompanied by the dissemination of production waste, possible industrial disasters caused by human factor, and/or malfunctioning of technical systems and technological processes, etc. Finally, the desired plan must be first and foremost feasible technically, economically and ecologically, and secondly, must be maximizing the expected profit, taking into account possible additional costs and compensation for damage caused by the danger of overflowing the special settling tank and the emission of part of its hazardous contents into the external environment (waste disposal costs; fines; elimination of the pollution consequences; etc.). Let us introduce the following notation for the parameters and variables of the studied problem, trying as far as possible to keep the notation introduced in the section 2 for the comparable parameters and variables of the general predictive-planning problem: $x \in R_{+}^{1}$ is the desired vector of the planned volume of biogas production, where $j$-th component $x_{j}(j=\overline{1, n})$ indicates the expected volume of $j$-th biogas component (methane, carbon dioxide, hydrogen, hydrogen sulphide, ammonia, nitrogen oxides, oxygen, etc.; for more detail, see [14], [15]); $y \in R_{+}^{1}$ is the desired predicted volume of the substance being discharged from the special settling tank; $y_{j}^{\max }(j=\overline{1, N})$ is a maximum allowable volume (for example, being defined following the regulatory requirements) of a substance emission from the special settling tank with or without taking into account the content of hazardous waste in the implementation of the $j$-th scenario $S_{j}$ of environmental conditions; $V$ is a volume of a special settling tank; $U_{j}(j=\overline{1, N})$ is a volume of external mass (for example, water) entering the special settling tank and mixing with its contents while implementing the $j$-th scenario $S_{j}$ of environmental conditions; $X \subset R_{+}^{n}$ is the set of technologically and economically feasible plans of biogas production; an objective function $w(x): X \rightarrow R^{1}$ describes a profit gained from biogas production without taking into account any additional costs and compensation for damage associated with the possible emission of part of the hazardous content from a special settling tank; function $f(x): X \rightarrow R_{+}^{1}$ describes the dynamics of the waste volume being stored in a special settling tank; function $f_{j}(x): X \rightarrow R_{+}^{1}, j=\overline{1, N}$ describes the spending focused on forced reduction of concentration $c_{j}(x)$ and volumes of harmful substances in a special settling tank while implementing the $j$-th scenario $S_{j}$ of environmental conditions; function $g_{j}(x): X \rightarrow R_{+}^{1}, j=\overline{1, N}$ describes the spending result $f_{j}(x)$ aimed at reduction of the waste volume in a special settling tank while implementing the $j$-th scenario $S_{j}$ of environmental conditions, $g_{j}(x)=g_{j}\left(f_{j}(x)\right)$; function $\varphi_{j}(y, x): Y \times X \rightarrow R_{+}^{1}, j=\overline{1, N}$ describes the overall ecological damage (as it has been mentioned above, damage is measured and is estimated in terms of the final result of biogas production, i.e. in profit) along with penalties and the costs of eliminating the consequences of the $j$-th scenario $S_{j}$ implementation of environmental conditions.

\subsection{Construction of the mathematical model}

The proposed mathematical model for the predictive-planning problem being formulated in subsection 3.1 is the following:

$$
\tilde{w}(x)=w(x)-\sum_{j=1}^{N} p_{j} \cdot\left(\varphi_{j}\left(y,\left.\frac{f(x)-g_{j}\left(f_{j}(x)\right)}{f(x)+U_{j}}\right|_{f(x)+U_{j}>V}\right)+f_{j}(x)\right) \rightarrow \max ,
$$




$$
\begin{gathered}
\left.\max \left\{f(x)+U_{j}-V ; 0\right\} \cdot \frac{f(x)-g_{j}\left(f_{j}(x)\right)}{f(x)+U_{j}}\right|_{f(x)+U_{j}>V}-y_{j}^{\max } \leq 0, \forall j=\overline{1, N}, \\
\left|y-\sum_{j=1}^{N} p_{j} \cdot \max \left\{f(x)+U_{j}-V ; 0\right\}\right| \leq \delta, \\
x \in X,
\end{gathered}
$$

where $\delta>0$ is a parameter, which indicates the range of possible deviations for the predicted volume of emissions depending on its mathematical expectation; notation of a kind $\left.a\right|_{b}$ means $\left.a\right|_{b}=a$ if $b$, and $\left.a\right|_{b}=0$ otherwise.

The following description of the mathematical model (6)-(9) allows, first, to understand the meaning of each of its components (i.e., the objective function and its constraints), and, secondly, to explain its obtaining process based on the qualitative model described in subsection 3.1 (the introduction of the required parameters, variables and functions for the studied problem and its detailed description). In (6), the maximized objective function $\tilde{w}(x)$ means the profit adjusted for the expected costs $\sum_{j=1}^{N} p_{j} \cdot\left(\varphi_{j}\left(y, c_{j}(x)\right)+f_{j}(x)\right)$, associated with a decrease in the volume of hazardous waste and the overflow of a special settling tank, where

$$
c_{j}(x)=\left\{\begin{array}{l}
\frac{f(x)-g_{j}\left(f_{j}(x)\right)}{f(x)+U_{j}} \text { if } f(x)+U_{j}>V, \\
0 \text { otherwise }
\end{array}\right.
$$

is the mean concentration of harmful substances in the emission from a special settling tank, while implementing the $j$-th scenario $S_{j}$ of environmental conditions. For $\forall j=\overline{1, N}$ constraint-inequality (7) is the upper limit on the amount of emission and/or on the content of harmful substances in it, while implementing the $j$-th scenario $S_{j}$ of environmental conditions. If to use the notation (10) and introduce a new notation

$$
y_{j}(x)=\max \left\{f(x)+U_{j}-V ; 0\right\},
$$

which denotes the dynamics of the substance volume being discharged from a special settling tank, while implementing the $j$-th scenario $S_{j}$ of environmental conditions and for the implementation of the selected plan $x \in X$, then (7) can be rewritten as $c_{j}(x) \cdot y_{j}(x) \leq y_{j}^{\max }, \forall j=\overline{1, N}$, which essence is quite obvious. Inequality (8), which taking into account the notation (11) has a simpler and more comprehensive form $\left|y-\sum_{j=1}^{N} p_{j} \cdot y_{j}(x)\right| \leq \delta$, is a constraint for desired plan $x$ and prediction $y$. This constraint is introduced in order to set an admissible set of predictions close to the expected value of the emission volume for the chosen plan $x$.

Finally, the restriction (9) is a natural requirement of the technological and economic admissibility of the desired plan for the production of biogas.

\section{Conclusions}

In this study:

1. There is formulated and formalized a predictive-planning problem in the form of general statement, as well as its mathematical model is constructed.

2. There is formalized a studied predictive-planning problem of biogas production from animal husbandry waste, and its qualitative model is constructed.

3. A single-criterion optimization model is proposed for the considered predictive-planning problem of biogas production from animal waste. 
4. The essence of each of the components of the proposed single-criterion optimization model is specified, which allows to understand and substantiate the transition from the constructed qualitative model to the proposed mathematical model.

\section{References}

[1] Evans G.M., Furlong J.C. Environmental Biotechnology: Theory and Application.Chichester: John Wiley \& Sons, 2003.xiii +285 p.

[2] Preliminary Impact Assessment for an Initiative on the Biological Treatment of Biodegradable Waste. Final Study Report, December 2004. [online] [28.03.2019]. Available at: http://ec.europa.eu/environment/waste/compost/pdf/ia_biowaste_directive_report.pdf

[3] Lemmes B. The "Tao" of organics, Wastes Management. The Monthly Journal of the Institute of Wastes Management, 1998, $18 \mathrm{p}$.

[4] Report on existing methods and good practices on household organic waste management and treatment, including large scale drying system "Development and demonstration of an innovative household dryer for the treatment of organic waste", DRYWASTE (LIFE 08 ENV/GR/000566). March 2011. [online] [28.03.2019]. Available at: http://www.uest.gr/drywaste/site/ Deliverable10.pdf

[5] Mavlyudova L.N. Development of technological solutions for the disposal of animal waste with the production of useful energy and fertilizers. Master's Thesis. Tolyatti: Tolyatti State University Press, 2017. 124 p.

[6] ForsterCh.F.,Wase D.A.J. Environmental Biotechnology. London: Ellis Horwood Publishing, 1987. $454 \mathrm{p}$.

[7] Lehninger A.L. Principles of Biochemistry. Volume 3. Moscow: MIR, 1985. 368 p. (in Russian).

[8] Ershov E.B., Levitin E.S. To the problem of the joint determination of the prognosis and plan in complex systems. In: Problems of Mathematical Programming, Moscow: Computing Center of the Russian Academy of Sciences, 1992, pp. 66-85.

[9] Levitin E.S. Optimization problems with extremal constraints. Part I: General concepts, formulation, and main problems. Automation and Remote Control, vol. 56, no. 7, 1995, pp. 907917. (in Russian).

[10]Levitin E.S. Optimization problems with extremal constraints. Part II: Application to mathematical problems of system analysis.- Automation and Remote Control, vol. 56, no. 12, 1995, pp. 1674-1686. (in Russian).

[11]Ershov E.B., Levitin E.S. On models of predictive-and-planning kind for solving social-andeconomic problems. Economics and Mathematical Methods, vol. 49.no. 2, 2013, pp. 3-18. (in Russian).

[12] Podberezkin A.I., Aleksandrov M.V., Borishpolets K.P., etc. Strategic prognostication and planning of foreign and defence policy.Volume 1: Theoretical foundations of the system of analysis, prognostication and planning of foreign and defence policy. Moscow: Moscow State Institute of International Relations, 2015. 796 p. (in Russian).

[13] Podberezkin A.I., Aleksandrov M.V., Borishpolets K.P., etc. Strategic prognostication and planning of foreign and defence policy.Volume 2: Prognostication of scenarios of the development of the international and military-and-political situations for the period up to 2050 . Moscow: Moscow State Institute of International Relations, 2015. 722 p. (in Russian).

[14] Baader W., Dohne E., Brenndorfer M. Biogas in Theory and Practice. Moscow: KOLOSS, 1982. 148 p. (in Russian).

[15] Alternative Fuels Data Center, U.S. Department of Energy: Energy Efficiency \& Renewable Energy.[online] [28.03.2019]. Available at: https://afdc.energy.gov/ 